\title{
Analysis of Discrete Adjoints for Upwind Numerical Schemes
}

\author{
Zheng Liu and Adrian Sandu \\ Department of Computer Science, Virginia Polytechnic Institute \\ and State University, Blacksburg, VA 24061 \\ \{liuzheng, sandu\}@vt.edu
}

\begin{abstract}
This paper discusses several aspects related to the consistency and stability of the discrete adjoints of upwind numerical schemes. First and third order upwind discretizations of the one-dimensional advection equation are considered in both the finite difference and finite volume formulations. We show that the discrete adjoints may lose consistency and stability near the points where upwinding is changed, and near inflow boundaries where the numerical scheme is changed. The impact of adjoint inconsistency and instability on data assimilation is analyzed.
\end{abstract}

\section{Introduction}

Adjoint models 3 are widely used in control and in data assimilation in conjunction with the optimization of large scale models. The adjoint methodology can efficiently provide gradients of objective functionals that are formulated in terms of the state of the model.

There are two ways to derive adjoint models 8 . The continuous approach solves numerically the adjoint equation derived from the forward model. The discrete approach formulates directly the adjoint of the forward numerical scheme. The discrete approach is highly attractive since the discrete adjoints, in principle, can be generated automatically [1].

Discrete adjoints of upwind numerical methods pose particular challenges. Symes and Sei 7] pointed out that the consistency of the numerical scheme is not automatically inherited by its discrete adjoint due to the upwind character of the forward scheme. Giles studied the construction and properties of discrete adjoints for hyperbolic systems with shocks [4,5]. Homescu and Navon [6] discuss the optimal control of flows with discontinuities.

In this paper we study the consistency and stability of discrete adjoints for upwind numerical schemes. The focus is on the advection equation

$$
\frac{\partial C}{\partial t}+\frac{\partial(U C)}{\partial x}=0
$$

and on the corresponding adjoint equation

$$
\frac{\partial \lambda}{\partial t}+U \frac{\partial \lambda}{\partial x}=0
$$


Advection is the prototype for hyperbolic problems requiring upwinding, and is in itself a fundamental model with many applications. Our discussion includes the case where the wind field has sources and sinks. This situation arises in the directional split solutions of multidimensional advection equation. The consideration of sources and sinks is also important for being able to later extend the results to nonlinear systems.

The paper is organized as follows. The consistency analysis in carried out in Section 2 and the stability analysis in Section 3. Numerical examples are provided in Section 4 and Section 5 summarizes the main findings of this work.

\section{Consistency Analysis}

In this section we consider several upwind discretizations of (1) and study the consistency of their discrete adjoint schemes with the continuous equation (2).

\subsection{First Order Finite Difference Scheme}

Forward Scheme. We start with the first order upwind discretization

$$
\begin{gathered}
C_{i}^{\prime}=(1 / \Delta x)\left[\gamma_{i}^{+} f_{i-1}+\left(\gamma_{i}^{-}-\gamma_{i}^{+}\right) f_{i}-\gamma_{i}^{-} f_{i+1}\right] \\
\gamma_{i}^{+}=1 \text { if } U_{i} \geq 0, \quad \gamma_{i}^{+}=0 \text { if } U_{i}<0, \quad \gamma^{-}=1-\gamma^{+}, \quad f_{i}=U_{i} C_{i} .
\end{gathered}
$$

The Dirichlet boundary conditions are $C_{0}=C_{L B}$ and $C_{N+1}=C_{R B}$. Here, and throughout the paper, $C^{\prime}$ denotes the time derivative of $C$.

Adjoint Scheme. The corresponding discrete adjoint is

$$
\lambda_{i}^{\prime}=\left(U_{i} / \Delta x\right)\left[-\gamma_{i-1}^{-} \lambda_{i-1}+\left(\gamma_{i}^{-}-\gamma_{i}^{+}\right) \lambda_{i}+\gamma_{i+1}^{+} \lambda_{i+1}\right]
$$

with the boundary conditions $\lambda_{0}=\lambda_{N+1}=0$.

Consistency inside the domain. If the wind direction is the same throughout the stencil $(i-1, i, i+1)$ then equation (4) is simply the first order spatial discretization method applied to (2). In this case consistency is assured.

We now consider the case where a shift in the wind direction occurs inside the stencil, e.g., $U_{i-1}<0$ and $U_{i}, U_{i+1} \geq 0$. The discrete adjoint (4) is:

$$
\lambda_{i}^{\prime}=\left(U_{i} / \Delta x\right)\left[-\lambda_{i-1}-\lambda_{i}+\lambda_{i+1}\right]
$$

The Taylor expansion of the above scheme around the $i^{\text {th }}$ node reveals that the the method is formally inconsistent with the continuous adjoint equation. The continuous wind velocity is small around the point of sign change, $U=$ $\mathcal{O}\left(\Delta x^{m}\right)$, and from (5) we have that $\lambda^{\prime}=\mathcal{O}\left(\Delta x^{m-1}\right)$. The consistency is maintained if $m>1$, and is lost for $m \leq 1$. The latter situation includes the most common situation were $U=\mathcal{O}(\Delta x)$, and also the case of a discontinuous wind field $U=\mathcal{O}(1)$. 
Consistency near the boundary. The adjoint inflow boundary condition for $U_{0}<$ 0 is $\lambda_{0}=0$. The discrete adjoint scheme near the boundary

$$
\lambda_{1}^{\prime}=\left(U_{1} / \Delta x\right)\left[-\gamma_{0}^{-} \lambda_{0}+\left(\gamma_{1}^{-}-\gamma_{1}^{+}\right) \lambda_{1}+\gamma_{2}^{+} \lambda_{2}\right]
$$

is consistent with the adjoint equation.

In conclusion the discrete adjoint of the first order upwind scheme is inconsistent near sources and sinks, and is consistent near the boundaries.

\subsection{Third Order Finite Difference Scheme}

Forward Scheme. We consider the third order biased upwind scheme:

$$
\begin{aligned}
C_{i}^{\prime}= & (1 / \Delta x)\left[-\frac{1}{6} \gamma_{i}^{+} f_{i-2}+\left(\frac{1}{3}+\frac{2}{3} \gamma_{i}^{+}\right) f_{i-1}+\frac{1}{2}\left(1-2 \gamma_{i}^{+}\right) f_{i}\right. \\
& \left.+\left(\frac{2}{3} \gamma_{i}^{+}-1\right) f_{i+1}+\frac{1}{6}\left(1-\gamma_{i}^{+}\right) f_{i+2}\right] \quad \text { for } i \geq 3, \\
C_{1}^{\prime}= & (1 / \Delta x)\left[\gamma_{1}^{+} f_{L B}+\left(\frac{3}{2}-\frac{5}{2} \gamma_{1}^{+}\right) f_{1}-2\left(1-\gamma_{1}^{+}\right) f_{2}+\frac{1}{2}\left(1-\gamma_{1}^{+}\right) f_{3}\right], \\
C_{2}^{\prime}= & (1 / \Delta x)\left[-\frac{1}{6} \gamma_{2}^{+} f_{L B}+\left(\frac{1}{3}+\frac{2}{3} \gamma_{2}^{+}\right) f_{1}\right. \\
& \left.+\left(\frac{1}{2}-\gamma_{2}^{+}\right) f_{2}+\left(\frac{2}{3} \gamma_{2}^{+}-1\right) f_{3}+\frac{1}{6}\left(1-\gamma_{2}^{+}\right) f_{4}\right] .
\end{aligned}
$$

The formulation corresponds to an inflow left boundary.

Adjoint Scheme. The corresponding discrete adjoint reads:

$$
\begin{aligned}
\lambda_{i}^{\prime}= & \left(U_{i} / \Delta x\right)\left[\frac{1}{6}\left(1-\gamma_{i-2}^{+}\right) \lambda_{i-2}+\left(\frac{2}{3} \gamma_{i-1}^{+}-1\right) \lambda_{i-1}\right. \\
& \left.+\left(\frac{1}{2}-\gamma_{i}^{+}\right) \lambda_{i}+\left(\frac{1}{3}+\frac{2}{3} \gamma_{i+1}^{+}\right) \lambda_{i+1}-\frac{1}{6} \gamma_{i+2}^{+} \lambda_{i+2}\right]
\end{aligned}
$$

Consistency inside the domain. The analysis is similar to the first order case. If the wind direction is the same throughout the stencil $(i-2, i-1, i, i+1, i+2)$ the equation (8) is consistent with the continuous adjoint. If the wind sign changes (i.e., there is a source or a sink) inside the stencil then $U=\mathcal{O}\left(\Delta x^{m}\right)$, and the scheme is inconsistent for $m \leq 1$.

Consistency near the boundary. The numerical scheme (7) shifts to a lower order, smaller stencil method near the boundaries. With an inflow left boundary the discrete adjoint equation for the first node is:

$$
\lambda_{1}^{\prime}=\left(U_{1} / \Delta x\right)\left[-\lambda_{1}+\lambda_{2}-\frac{1}{6} \lambda_{3}\right]
$$

This should reflect an outflow condition for the adjoint. However (9) is inconsistent with the continuous equation (2). 


\subsection{Finite Volume Approach}

We have also studied the consistency of adjoints based on forward finite volume schemes of the form

$$
C_{i}^{\prime}=\frac{F_{i-\frac{1}{2}}-F_{i+\frac{1}{2}}}{\Delta x} .
$$

Specifically, we considered first order fluxes for a staggered grid discretization

$$
F_{i-\frac{1}{2}}=U_{i-\frac{1}{2}}^{+} C_{i-1}+U_{i-\frac{1}{2}}^{-} C_{i}
$$

as well as third order fluxes

$$
\begin{aligned}
F_{i-\frac{1}{2}}= & -\frac{1}{6} U_{i-\frac{1}{2}}^{+} C_{i-2}+\left(\frac{5}{6} U_{i-\frac{1}{2}}^{+}+\frac{1}{3} U_{i-\frac{1}{2}}^{-}\right) C_{i-1} \\
& +\left(\frac{1}{3} U_{i-\frac{1}{2}}^{+}+\frac{5}{6} U_{i-\frac{1}{2}}^{-}\right) C_{i}-\frac{1}{6} U_{i-\frac{1}{2}}^{-} C_{i+1} \text { for } i \geq 3 \\
F_{\frac{1}{2}}= & U_{\frac{1}{2}}^{+} C_{L B}+\frac{3}{2} U_{\frac{1}{2}}^{-} C_{1}-\frac{1}{2} U_{\frac{1}{2}}^{-} C_{2} \\
F_{\frac{3}{2}}= & \left(\frac{1}{2} U_{\frac{3}{2}}^{+}+\frac{1}{3} U_{\frac{3}{2}}^{-}\right) C_{1}+\left(\frac{1}{2} U_{\frac{3}{2}}^{+}+\frac{5}{6} U_{\frac{3}{2}}^{-}\right) C_{2}-\frac{1}{6} U_{\frac{3}{2}}^{-} C_{3}
\end{aligned}
$$

A similar analysis revealed that: (1) the discrete adjoint of the first order finite volume scheme is consistent with the adjoint equation except for the points where there is a sink or source; (2) the discrete adjoint of the first order finite volume scheme is consistent at the boundaries; (3) the discrete adjoint of the third order finite volume scheme is consistent except for the case when there are sinks or sources of velocity within the stencil (when it becomes inconsistent); and (4)the discrete adjoint of the third order finite volume scheme is not consistent at nodes near the inflow boundary.

\section{$3 \quad$ Stability Analysis}

In this section the von Neumann stability analysis of the discrete adjoints is carried out for the finite difference formulations.

The first order spatial discretization leads to the system (3) of ordinary differential equations, which is resolved in time using the forward Euler method. The Courant-Friedrichs-Levy (CFL) stability condition for the fully discrete forward scheme is $\sigma \leq 1$, where $\sigma$ is the Courant number. The adjoint of the fully discrete scheme is the equation (4) resolved in time with the forward Euler method.

The system of ordinary differential equations resulting from the third order spatial discretization (7) is resolved in time with the strongly stable, two stage, second order explicit Runge-Kutta method

$$
C^{\alpha}=C^{n}+A C^{n}, \quad C^{\beta}=C^{\alpha}+A C^{\alpha}, \quad C^{n+1}=\frac{1}{2}\left(C^{n}+C^{\beta}\right) .
$$

The CFL stability condition for the fully discrete forward scheme is $\sigma \leq 0.87$. The corresponding discrete adjoint is the equation (8), resolved in time with the same Runge-Kutta scheme (13). 
We are concerned with the stability of the resulting discrete adjoints. In the interest of brevity we omit the derivation and present directly the results. The von Neumann stability analysis reveals the dependency of the amplification factors on the Courant number $(\sigma)$ and on the wave number $(\theta=k \Delta x /(2 \pi))$. Fig. 1 presents the areas of stability (white) and instability (gray) in the $(\sigma, \theta)$ plane for different situations.

The stability of the first order discrete adjoint is the same as for the forward method when all wind directions in the stencil $(i-1, i, i+1)$ are the same. However, as seen in Fig. 1(a), when the wind changes direction inside the stencil a stricter stability condition $(\sigma \leq 0.4)$ is required.

Fig. 1(b) reveals that the stability of the third order discrete adjoint is the same as for the forward method when the wind does not change direction within the stencil $(i-2, \cdots, i+2)$. Fig. 11(d) shows that the discrete adjoint is stable with a slightly restricted Courant number if the wind is negative in the leftmost two stencil points. An interesting situation arises when the wind is negative in only the leftmost grid point. In this case the discrete adjoint is unconditionally unstable as seen in Fig. 11(c).

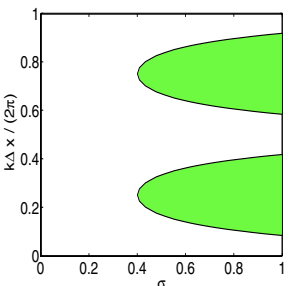

(a) $1^{\text {st }}$ order $U_{i-1}<0$ $U_{i}, U_{i+1} \geq 0$

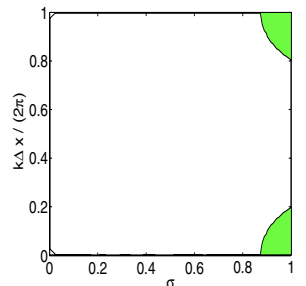

(b) $3^{\text {rd }}$ order

$U_{i-2} \cdots U_{i+2} \geq 0$

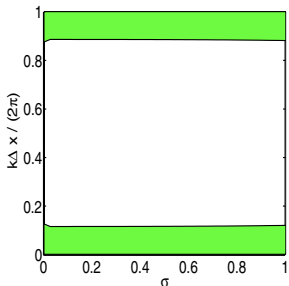

(c) $3^{\text {rd }}$ order $U_{i-2}<0$; $U_{i-1} \cdots U_{i+2} \geq 0$

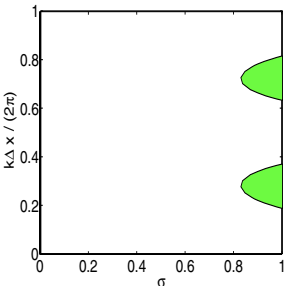

(d) $3^{\text {rd }}$ order $U_{i-2}, U_{i-1}<0$; $U_{i} \cdots U_{i+2} \geq 0$

Fig. 1. The stable (white) and unstable (gray) regions of the discrete adjoints in the Courant number $(\sigma)$ - wave number $(\theta=k \Delta x /(2 \pi))$ plane

\section{$4 \quad$ Numerical Examples}

To illustrate the inconsistency we use the discrete adjoint method to solve the adjoint equation. The initial condition is a sine function, and the numerical adjoint solutions after backward integration are shown in Fig. 2.

First we consider a linear wind field that has negative values in left half and positive values in the right half of the domain. The center is a source with zero velocity and both boundaries are outflow boundaries. Both the first order (Fig. 2(a)) and third order discrete adjoint solutions (Fig. 2(b)) are corrupted by the inconsistency near the central source. 
Next we consider a linear wind field with opposite sign. The center of the domain is now a sink with zero velocity and both boundaries are inflow boundaries. Both the first order (Fig. 2(c)) and third order discrete adjoint solutions (Fig. 2(d)) are corrupted by the inconsistency near the central sink. Moreover, the third order discrete adjoint is inconsistent near the boundaries (Fig. 2(d)) .

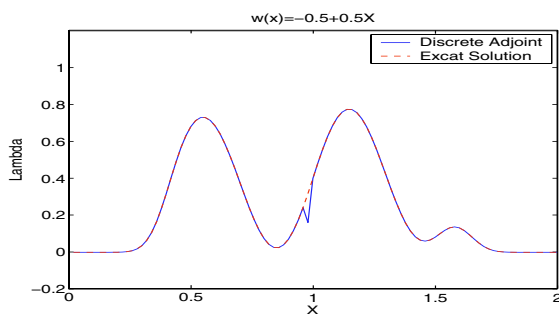

(a) $1^{\text {st }}$ order; $U(x)=0.5 x-0.5$

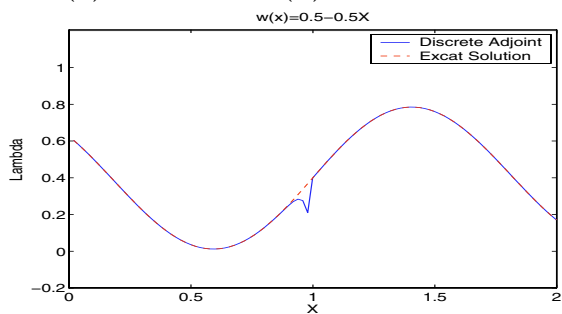

(c) $1^{\text {st }}$ order; $U(x)=-0.5 x+0.5$

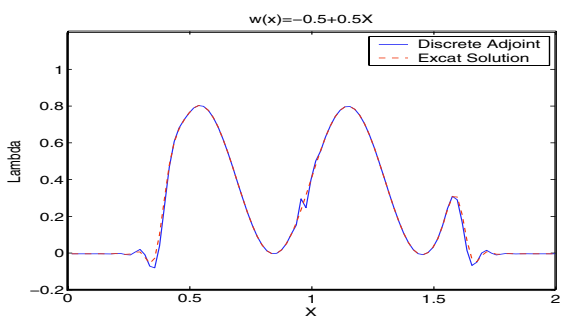

(b) $3^{\text {rd }}$ order; $U(x)=0.5 x-0.5$

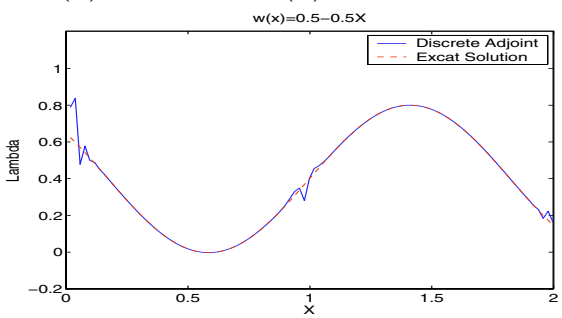

(d) $3^{\text {rd }}$ order; $U(x)=-0.5 x+0.5$

Fig. 2. The discrete adjoint solutions is not consistent with the continuous adjoint near sources/sinks. In addition, the third order solution is also inconsistent near inflow boundaries

We now assess the influence of inconsistency and instability on variational data assimilation. The limited-memory quasi-Newton optimization algorithm L-BFGS [2] is used to recover the initial conditions from observations of the solution at selected grid points and time moments. Assimilation experiments (not reported here) revealed that the convergence rates of the optimization process are nearly identical with discrete and with continuous adjoints as long as the Courant number is small. Consequently the inconsistency and instability of the discrete adjoints do not seem to impact visibly the data assimilation process.

The results of several assimilation experiments are shown in Fig. 3. Fig. 3(a) corresponds to a constant, positive wind field. The inflow (left) boundary adjoint inconsistency does not affect the optimization and the recovered initial condition matches well the reference. Fig. 3 (b) is for a piecewise constant wind field, with a source in the center and two outflow boundaries. The recovery near the source is good, however the recovery near the boundaries is poor. This is due to the information loss when the "particles" exit the domain. Fig. 3(c) considers a linear wind field, with a source of zero velocity in the center and two outflow 


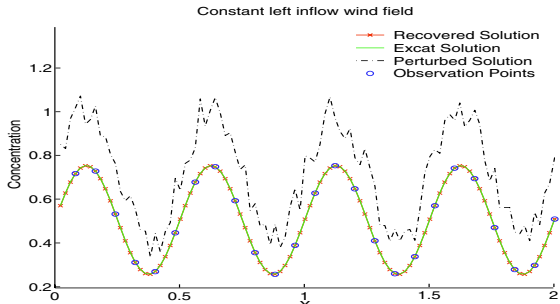

(a)

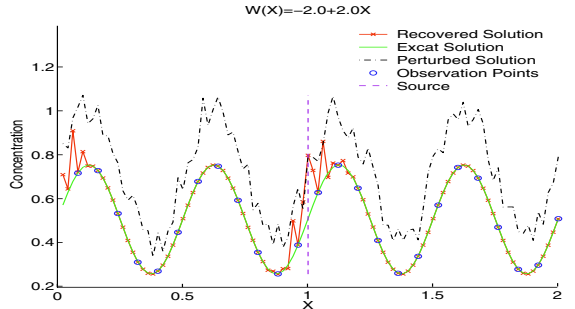

(c)

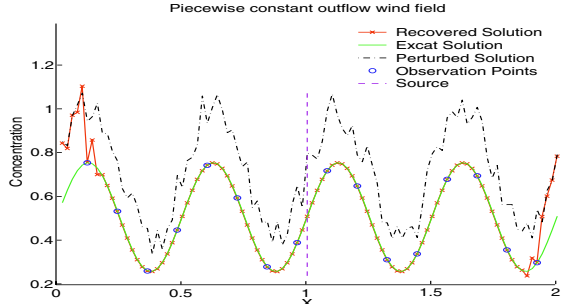

(b)

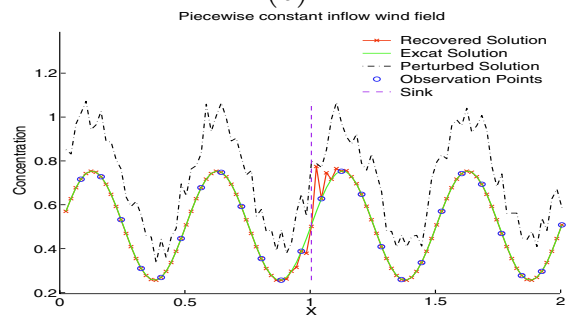

(d)

Fig. 3. (a) Constant, positive wind field; (b) Piecewise constant wind field, with a source in the center and two outflow boundaries; (c) Linear wind field, with a source of zero velocity in the center and two outflow boundaries; (d) Piecewise constant wind field, with a sink in the center and two inflow boundaries

boundaries. The recovery of the left outflow boundary is inaccurate, as expected. The recovery of the right outflow boundary is accurate due to the observation point placed on the boundary grid point. The error in recovered initial condition near the center is due to the very small wind speed. The "particles" cannot reach the nearest observation point during the simulation, which results in information loss. Fig. 3. (d) is for piecewise constant wind field, with a sink in the center and two inflow boundaries. The recovery error near the center is due to the information loss when the "particles" disappear into the sink (note that there is no observation point at the sink). Both sinks and sources lead to inconsistent discrete adjoints, however the recovery of the initial condition is difficult only for the sinks. Consequently, the recovery error is due to information loss and not to inconsistency.

\section{Conclusions}

In this paper we analyze the consistency and stability of discrete adjoints for upwind numerical schemes. The focus is on first and third order upwind discretizations of the one-dimensional advection equation.

The discrete adjoints are inconsistent with the continuous adjoint equation at inflow boundaries and near sinks or sources (i.e., points where the wind field changes sign). The von Neumann stability of the forward numerical scheme is 
not automatically maintained by the adjoint operation. Depending on the upwinding direction of different points inside the stencil the discrete adjoint can be: (1) linearly stable with a CFL time step restriction similar to that of the forward method, (2) linearly stable under a more stringent CFL condition, or (3) unconditionally unstable at a given point near sources or sinks.

The inconsistency and instability do not affect the performance of the optimization procedure in the data assimilation examples considered here. Both discrete and continuous adjoints lead to similar convergence rates for the recovery of the initial conditions. However, the optimization process is hindered by the loss of information occurring when: (1) the solution collapses into a sink or a shock; (2) the solution exits the domain through an outflow boundary; and (3) the solution features propagate only on a short distance, insufficient to reach one of the observation sites.

\section{Acknowledgements}

This work was supported by the National Science Foundation through the awards NSF CAREER ACI 0093139 and NSF ITR AP\&IM 0205198.

\section{References}

1. Evaluating derivatives: Principles and Techniques of Algorithmic Differentiation, volume 41 of Frontiers in Applied Mathematics. SIAM, 2000.

2. R. Byrd, P. Lu, and J. Nocedal. A limited memory algorithm for bound constrained optimization. SIAM Journal of Scientific and Statistical Computing, 16(5):11901208, 1995.

3. D. G. Cacuci. Sensitivity theory for nonlinear systems. I. nonlinear functional analysis approach. Journal of Mathematical Physics, 22:2794-2802, 1981.

4. M.B. Giles. Discrete adjoint approximations with shocks. Technical Report 02/10, Oxford University Computing Laboratory, Numerical Analysis Group, 2002.

5. M.B. Giles, M.C. Duta, and N.A. Pierce. Algorithm developments for discrete adjoint methods. AIAA Journal, 41(2):198-205, Feburary 2003.

6. C. Homescu and I.M. Navon. Optimal control of flow with discontinuities. Journal of Computational Physics, 187:660-682, 2003.

7. A. Sei and Symes W. A note on consistency and adjointness of numerical schemes. Technical Report CSRPC-TR95527, Center for Research in Parallel Computation, Rice University, January 1995.

8. Z. Sirkes and E. Tziperman. Finite difference of adjoint or adjoint of finite difference? Monthly Weather Review, 49:5-40, 1997. 